Research Article

\title{
Existence and Uniqueness Results of Volterra-Fredholm Integro-Differential Equations via Caputo Fractional Derivative
}

\author{
Ameth Ndiaye $\mathbb{D}^{1}$ and Fulgence Mansal $^{2}$ \\ ${ }^{1}$ Département de Mathématiques, FASTEF, UCAD, Dakar, Senegal \\ ${ }^{2}$ Département de Mathématiques et Informatique, UCAO, Dakar, Senegal \\ Correspondence should be addressed to Ameth Ndiaye; ameth1.ndiaye@ucad.edu.sn
}

Received 11 May 2021; Accepted 11 August 2021; Published 20 August 2021

Academic Editor: Ali Jaballah

Copyright (c) 2021 Ameth Ndiaye and Fulgence Mansal. This is an open access article distributed under the Creative Commons Attribution License, which permits unrestricted use, distribution, and reproduction in any medium, provided the original work is properly cited.

\begin{abstract}
In this paper, we study a Volterra-Fredholm integro-differential equation. The considered problem involves the fractional Caputo derivatives under some conditions on the order. We prove an existence and uniqueness analytic result by application of the Banach principle. Then, another result that deals with the existence of at least one solution is delivered, and some sufficient conditions for this result are established by means of the fixed point theorem of Schaefer. Ulam stability of the solution is discussed before including an example to illustrate the results of the proposal.
\end{abstract}

\section{Introduction}

Fractional calculus and differential equations of fractional order are of great importance since they can be used in analyzing and modeling real word phenomena [1-3]. Recently, there has been a very important progress in the study of the theory of differential equations of fractional order. The theory of differential equations of arbitrary order has been recently proved to be an important tool for modeling many physical phenomena. For more details, refer to [4-9].

The fractional integro-differential equations have been recently used as effective tools in the modeling of many phenomena in various fields of applied sciences and engineering such as acoustic control, signal processing, electrochemistry, viscoelasticity, polymer physics, electromagnetics, optics, medicine, economics, chemical engineering, chaotic dynamics, and statistical physics (see [10-16]).
Hattaf in [17] proposed a new definition of fractional derivative that generalizes the fractional derivatives $[18,19]$ with nonsingular kernel for both Caputo and Riemann-Liouville types.

The efficient numerical method based on a novel shifted piecewise cosine basis for solving Volterra-Fredholm integral equations of the second kind is investigated (see [20]).

Recently, Wang et al. in [21] studied a nonlinear fractional differential equations with Hadamard derivative and Ulam stability in the weighted space of continuous functions. Some sufficient conditions for existence of solutions are given by using fixed point theorems via a prior estimation in the weighted space of the continuous functions. Ahmad et al. in [22] discussed the existence of solutions for an initial value problem of nonlinear hybrid differential equations of Hadamard type.

Ahmed et al. [23] discussed the existence of solutions by means of endpoint theory for initial value problem of 
Hadamard and Riemann-Liouville fractional integro-differential inclusion of the form as follows:

$$
\left\{\begin{array}{l}
D^{\alpha}\left(x(t)-\sum_{i=1}^{m} I^{\rho_{i}} G_{i}(t, x(t)) \in F(t, x(t)), \quad t \in J=[1, T], 0<\alpha \leq 1,\right. \\
x(1)=0,
\end{array}\right.
$$

where $D^{\alpha}$ denotes the Hadamard fractional derivative of order $\alpha$ for $0<\alpha \leq 1$. $I^{\varphi}$ is the Riemann-Liouville integral of order $\varphi>0, \varphi \in\left\{\rho_{1}, \rho_{2}, \ldots, \rho_{m}\right\}, G_{i} \in C(J \times \mathbb{R}, \mathbb{R})$ with $G_{i}(1,0)=0$ for $i=1,2,3 \ldots, m$.

Very recent work like Hamoud et al. [24] established some new conditions for the existence and uniqueness of solutions for a class of nonlinear Hadamard fractional Volterra-Fredholm integro-differential equations with initial conditions. The homotopy perturbation method has been successfully applied to find the approximate solution of a Caputo fractional Volterra-Fredholm integro-differential equation.

Motivated by the above works, we will study the following problem of fractional integro-differential equations in the context of Caputo fractional derivative called Caputo fractional Volterra-Fredholm integro-differential equations of the form as follows:

$$
\left\{\begin{array}{l}
D^{\alpha}\left(x(t)-\sum_{i=1}^{m} I^{\rho_{i}} f_{i}(t, x(t))\right)=g(t, x(t), K x(t), H x(t)), \quad t \in J=[0,1] \\
x(0)=0 \\
D^{\alpha} x(0)=\eta, 1<\alpha<2
\end{array}\right.
$$

where $D^{\alpha}$ is in the sense of Caputo, $f: J \times \mathbb{R}^{n} \times \mathbb{R}^{n} \longrightarrow \mathbb{R}^{n}$ is a given function, $K$ and $H$ are linear integral operators defined by $K x(t)=\int_{0}^{t} k(t, \tau) x(t) \mathrm{d} \tau$ and $H x(t)=\int_{0}^{t} h$ $(t, \tau) x(t) \mathrm{d} \tau$, and its called Volterra-Fredholm integro-differential with $\theta_{1}=\sup \{|k(t, \tau)|:(t, \tau) \in J \times J\} \quad$ and $\theta_{2}=\sup \{|h(t, \tau)|:(t, \tau) \in J \times J\}$.

The paper is organised as follows. In Section 2, we recall some definitions and lemmas that are used for the proof of our main results. In Section 3, we prove the main theorems of this paper by the existence and uniqueness of the solution which have been proved and some numerical simulation of the solution. A brief conclusion is given in Section 6 .

\section{Preliminaries}

In this section, we introduce some definitions, lemmas, and preliminaries facts which are used throughout this paper (see [7] for more information). Let $|$.$| be a suitable norm in \mathbb{R}^{n}$ and $\|$.$\| be the matrix norm. Let E=C(J, \mathbb{R})$ denote the Banach space of continuous function on $J$ with the norm

$$
\|x\|=\sup \{|x|, x \in J\} .
$$

Definition 1. The Riemann-Liouville integral of order $\alpha>0$ for a continuous function $\varphi \in L^{1}((0,1], \mathbb{R})$ is given by

$$
I^{\alpha} \varphi(t)=\frac{1}{\Gamma(\alpha)} \int_{0}^{t}(t-\tau)^{\alpha-1} \varphi(t) \mathrm{d} \tau, \quad \forall t \in(0,1],
$$

with $\Gamma(\alpha)$ : $=\int_{0}^{\infty} e^{-u} u^{\alpha-1} \mathrm{~d} u$.

Definition 2. If $\varphi \in C^{n}([0,1], \mathbb{R})$ and $n-1<\alpha \leq n$, then the Caputo fractional derivative is given by

$$
\begin{aligned}
D^{\alpha} \varphi(t) & =I^{n-\alpha} \frac{\mathrm{d}^{n}}{\mathrm{~d} t^{n}}(\varphi(t)) \\
& =\frac{1}{\Gamma(n-\alpha)} \int_{0}^{t}(t-s)^{n-\alpha-1} \varphi^{(n)}(s) \mathrm{d} s,
\end{aligned}
$$

where the parameter $\alpha$ is the order of the derivative and is allowed to be real or even complex.

Lemma 1. Let $n \in \mathbb{N}^{*}$ and $n-1<\alpha<n$, then the general solution of $D^{\alpha} u(t)=0$ is given by

$$
u(t)=\sum_{i=0}^{n-1} c_{i} t^{i}
$$

such that $c_{i} \in \mathbb{R}, i=0,1,2, \ldots, n-1$.

Lemma 2. Taking $n \in \mathbb{N}^{*}$ and $n-1<\alpha<n$, then we have

$$
I^{\alpha} D^{\alpha} u(t)=u(t)+\sum_{k=0}^{n-1} \frac{u^{(k)}(0)}{k !} t^{k}
$$

with $t>0, n-1<\alpha<n$.

Definition 3. Let $X$ be a Banach space. Then, a map $T: X \longrightarrow X$ is called a contraction mapping on $X$ if there exists $q \in[0,1)$ such that

$$
\|T(x)-T(3 y)\| \leq q\|x-y\|
$$

for all $x, y \in X$.

Theorem 1 (Banach's fixed point theorem, see [25]). Let $\Omega$ be a nonempty closed subset of a Banach space $X$. Then, any 
contraction mapping $T$ of $\Omega$ into itself has a unique fixed point.

Theorem 2 (Schaefer's fixed point theorem, see [25]). Let $X$ be a Banach space, and let $N: X \longrightarrow X$ be a completely continuous operator. If the set $E=\{y \in X: y=\lambda N y$ for some $\lambda \in(0,1)\}$ is bounded, then $N$ has fixed points.

\section{Existence and Uniqueness Results}

We begin this section by some result that helps us for solving the problem considered in (2).

Lemma 3. Let $1<\alpha<2$ and $G \in C\left(J, \mathbb{R}^{n}\right)$. Then, we can state that the problem

$$
\left\{\begin{array}{l}
D^{\alpha}\left(x(t)-\sum_{i=1}^{m} I_{i}^{p} f_{i}(t, x(t))\right)=G(t), \quad t \in J=[0,1], \\
x(0)=0, D^{\alpha} x(0)=\eta,
\end{array}\right.
$$

admits as integral solution the following representation:

$$
x(t)=\frac{1}{\Gamma(\alpha)} \int_{0}^{t}(t-\tau)^{\alpha-1} G(\tau) \mathrm{d} \tau+\sum_{i=1}^{m} I^{\rho_{i}} f_{i}(t, x(t)) .
$$

Proof. Using Lemma 2, we get

$$
\begin{aligned}
& I^{\alpha} D^{\alpha}\left(x(t)-\sum_{i=1}^{m} I_{i}^{p} f_{i}(t, x(t))\right)=I^{\alpha} G(t), \\
& x(t)-\sum_{i=1}^{m} I_{i}^{p} f_{i}(t, x(t))=I^{\alpha} G(t)+c_{1} t+c_{0}, \\
& x(t)=I^{\alpha} G(t)+\sum_{i=1}^{m} I_{i}^{p} f_{i}(t, x(t))+c_{1} t+c_{0} .
\end{aligned}
$$

Using the initial conditions $x(0)=0$ and $D^{\alpha} x(0)=\eta$, we get $c_{1}=c_{2}=0$ which implies that the proof is completed.
Let us now transform the above problem to a fixed point one. Consider the nonlinear operator $T: E \longrightarrow E$ defined by

$$
\begin{aligned}
T x(t)= & \frac{1}{\Gamma(\alpha)} \int_{0}^{t}(t-\tau)^{\alpha-1} g(\tau, x(\tau), K x(\tau), H x(\tau)) \mathrm{d} \tau \\
& +\sum_{i=1}^{m} I^{\rho_{i}} f_{i}(t, x(t)) .
\end{aligned}
$$

To prove the main results, we need to work with the following hypotheses:

(H1) There exists a constant $L_{f}>0$, such that

$$
\left|f_{i}(t, x(t))-f_{i}(t, y(t))\right| \leq L_{f}|x(t)-y(t)| .
$$

(H2) There exist functions $c_{1}(t), c_{2}(t), c_{3}(t)$, and $a_{i}(t) \in C(J, \mathbb{R})$ such that

$$
\begin{aligned}
& |g(t, x, y, z)| \leq c_{1}(t)+c_{2}(t)|y|+c_{2}(t)|z|, \quad \forall(t, x, y, z) \in I \times \mathbb{R}^{3}, \\
& \left|f_{i}(t, x)\right| \leq a_{i}(t), \quad \forall(t, x) \in I \times \mathbb{R} .
\end{aligned}
$$

Set $\sup _{i \in I}\left|c_{1}(t)\right|=\left\|c_{1}\right\|, \sup _{i \in I}\left|c_{2}(t)\right|=\left\|c_{2}\right\|, \sup _{i \in I} \mid c_{3}$ $(t) \mid=\left\|c_{3}\right\|$, and $\sup _{i \in I}\left|a_{i}(t)\right|=\left\|a_{i}\right\|, i=1, \ldots, m$.
(H3) There exist constants $L_{1}, L_{2}, L_{3}>0$ such that

$$
\left|g\left(t, x_{1}, y_{1}, z_{1}\right)-g\left(t, x_{2}, y_{2}, z_{2}\right)\right| \leq L_{1}\left|x_{1}-x_{2}\right|+L_{2}\left|y_{1}-y_{2}\right|+L_{3}\left|z_{1}-z_{2}\right|, \quad \forall t \in J, x_{i}, y_{i}, z_{i} \in \mathbb{R}, i=1,2
$$

Also, we consider the quantity:

$$
R=\frac{L_{1}}{\Gamma(\alpha+1)}+\frac{\theta_{1} L_{2}+\theta_{2} L_{3}}{\Gamma(\alpha)}+\sum_{i=1}^{m} \frac{L_{f}}{\Gamma\left(\rho_{i}+1\right)} .
$$

Theorem 3. Assume that the hypothesis (H1)-(H2) are fulfilled, and if

$$
L_{f} \sum_{i=1}^{m} \frac{1}{\Gamma\left(\rho_{i}+1\right)}<1
$$


then there exists at least one solution for the problem (2).

Proof. Consider the ball $B_{r}=\{x \in E$ : $\|x\| \leq r\}$ with $r>0$, where

$$
r \geq \frac{\sum_{i=1}^{m}\left(\left\|a_{i}\right\| / \Gamma\left(\rho_{i}+1\right)\right)+\left(\left\|c_{1}\right\| / \Gamma(\alpha+1)\right)}{1-(1 / \Gamma(\alpha))\left(\left\|c_{2}\right\| \theta_{1}+\left\|c_{3}\right\| \theta_{2}\right)} .
$$

We define the operators $P$ and $Q$ such that $T=P+Q$, by

$$
P x(t)=\frac{1}{\Gamma(\alpha)} \int_{0}^{t}(t-\tau)^{\alpha-1} g(\tau, x(\tau), K x(\tau), H x(\tau)) \mathrm{d} \tau
$$$$
Q x(t)=\sum_{i=1}^{m} \frac{1}{\Gamma\left(\rho_{i}\right)} \int_{0}^{t}(t-\tau)^{\rho_{i}-1} f_{i}(t, x(t)) \mathrm{d} \tau \text {. }
$$

For any $x \in B_{r}$, we have

$$
\begin{aligned}
|T x(t)| \leq & \frac{1}{\Gamma(\alpha)} \int_{0}^{t}(t-\tau)^{\alpha-1}|g(\tau, x(\tau), K x(\tau), H x(\tau))| \mathrm{d} \tau+\sum_{i=1}^{m} \frac{1}{\Gamma\left(\rho_{i}\right)} \int_{0}^{t}(t-\tau)^{\rho_{i}-1}\left|f_{i}(\tau, x(\tau))\right| \mathrm{d} \tau \\
\leq & \frac{1}{\Gamma(\alpha)} \int_{0}^{t}(t-\tau)^{\alpha-1}\left(\left|c_{1}(\tau)\right|+\left|c_{2}(\tau)\right||K x(\tau)|+\left|c_{3}(\tau)\right||H x(\tau)|\right) \mathrm{d} \tau \\
& +\sum_{i=1}^{m} \frac{1}{\Gamma\left(\rho_{i}\right)} \int_{0}^{t}(t-\tau)^{\rho_{i}-1}\left|a_{i}(\tau)\right| \mathrm{d} \tau \\
\leq & \frac{\left\|c_{1}\right\|}{\Gamma(\alpha+1)}+\left(\left\|c_{2}\right\| \theta_{1}+\left\|c_{3}\right\| \theta_{2}\right) \frac{r}{\Gamma(\alpha)}+\sum_{i=1}^{m} \frac{\left\|a_{i}\right\|}{\Gamma\left(\rho_{i}+1\right)},|\operatorname{Px}(t)+Q x(t)| \leq r .
\end{aligned}
$$

Now, we will show that $P$ is continuous and compact. The operator $P$ is obviously continuous. Also, $P$ is uniformly bounded on $B_{r}$ as

$$
\|P x\| \leq \frac{\left\|c_{1}\right\|}{\Gamma(\alpha+1)}+\left(\left\|c_{2}\right\| \theta_{1}+\left\|c_{3}\right\| \theta_{2}\right) \frac{r}{\Gamma(\alpha)} .
$$

$$
\begin{aligned}
\left|P x\left(t_{2}\right)-P x\left(t_{1}\right)\right|= & \frac{1}{\Gamma(\alpha)} \int_{0}^{t_{2}}\left(t_{2}-\tau\right)^{\alpha-1} g(\tau, x(\tau), K x(\tau), H x(\tau)) \mathrm{d} \tau \\
& -\frac{1}{\Gamma(\alpha)} \int_{0}^{t_{1}}\left(t_{1}-\tau\right)^{\alpha-1} g(\tau, x(\tau), K x(\tau), H x(\tau)) \mathrm{d} \tau, \\
\left|P x\left(t_{2}\right)-P x\left(t_{1}\right)\right| \leq & \frac{\left\|c_{1}\right\|+\left\|c_{2}\right\| \theta_{1}+\left\|c_{3}\right\| \theta_{2}}{\gamma(\alpha+1)}\left(t_{2}^{\alpha}-t_{1}^{\alpha}\right) .
\end{aligned}
$$

We remark that when $t_{2} \longmapsto t_{1}$, the quantity $\left\|P x\left(t_{2}\right)-P x\left(t_{1}\right)\right\| \longmapsto 0$.
Thus, $P$ is equicontinuous and relatively compact on $B_{r}$. Then, we show by the Arzelà-Ascoli theorem that $P$ is 
compact on $B_{r}$. Let us show now that $Q$ is a contraction mapping and consider $x, y \in B_{r}$.
Then, for $t \in J$, we have

$$
\begin{aligned}
|Q x(t)-Q y(t)| & =\left|\sum_{i=1}^{m} \frac{1}{\Gamma\left(\rho_{i}\right)} \int_{0}^{t}(t-\tau)^{\rho_{i}-1} f_{i}(\tau, x(\tau)) \mathrm{d} \tau-\sum_{i=1}^{m} \frac{1}{\Gamma\left(\rho_{i}\right)} \int_{0}^{t}(t-\tau)^{\rho_{i}-1} f_{i}(\tau, y(\tau)) \mathrm{d} \tau\right| \\
& \leq \sum_{i=1}^{m} \frac{1}{\Gamma\left(\rho_{i}\right)} \int_{0}^{t}(t-\tau)^{\rho_{i}-1}\left|f_{i}(\tau, x(\tau))-f_{i}(\tau, y(\tau))\right| \mathrm{d} \tau \\
& \leq \sum_{i=1}^{m} \frac{1}{\Gamma\left(\rho_{i}\right)} \int_{0}^{t}(t-\tau)^{\rho_{i}-1} L_{f}\|x-y\| \\
& \leq L_{f} \sum_{i=1}^{m} \frac{1}{\Gamma\left(\rho_{i}+1\right)}\|x-y\| .
\end{aligned}
$$

We can therefore deduce that $T$ is a contraction map. Since all the assumptions of the Krasnoselskii fixed point $r \geq \frac{(\mu / \Gamma(\alpha+1))+\sum_{i=1}^{m}\left(\nu_{i} / \Gamma\left(\rho_{i}+1\right)\right)}{\left(L_{1} / \Gamma(\alpha+1)\right)+\left(\theta_{1} L_{2}+\theta_{2} L_{3} / \gamma(\alpha)\right)+L_{f} \sum_{i=1}^{m}\left(1 / \Gamma\left(\rho_{i}+1\right)\right)}$. theorem are now satisfied, problem (2) then admits at least one solution on $J$ which ends the proof.

Let us set now

$$
\begin{aligned}
& \mu=\sup _{i \in J}|g(t, 0,0,0)|, \\
& v_{i}=\sup _{i \in J}\left|f_{i}(t, 0)\right| .
\end{aligned}
$$

Proof. We show that $T$ has a unique fixed point, which is unique solution of problem (2).

Our objective is to show that $T B_{r} \subset B_{r}$.

For $x \in B_{r}$, we have

Let $B_{r}=\{x \in E:\|x\| \leq r\}$ with $r>0$, where

$$
\begin{aligned}
|T x(t)|= & \left|\frac{1}{\Gamma(\alpha)} \int_{0}^{t}(t-\tau)^{\alpha-1} g(\tau, x(\tau), K x(\tau), H x(\tau)) \mathrm{d} \tau+\sum_{i=1}^{m} \frac{1}{\Gamma\left(\rho_{i}\right)} \int_{0}^{t}(t-\tau)^{\rho_{i}-1} f_{i}(\tau, x(\tau)) \mathrm{d} \tau\right| \\
\leq & \sup _{t \in J}\left[\frac{1}{\Gamma(\alpha)} \int_{0}^{t}(t-\tau)^{\alpha-1}|g(\tau, x(\tau), K x(\tau), H x(\tau))| \mathrm{d} \tau+\sum_{i=1}^{m} \frac{1}{\Gamma\left(\rho_{i}\right)} \int_{0}^{t}(t-\tau)^{\rho_{i}-1}\left|f_{i}(\tau, x(\tau))\right| \mathrm{d} \tau\right] \\
\leq & \sup _{t \in J}\left\{\frac{1}{\Gamma(\alpha)} \int_{0}^{t}(t-\tau)^{\alpha-1}|g(\tau, x(\tau), K x(\tau), H x(\tau))-g(\tau, 0,0,0)| \mathrm{d} \tau\right\} \\
& +\sum_{i=1}^{m} \frac{1}{\Gamma\left(\rho_{i}\right)} \int_{0}^{t}(t-\tau)^{\rho_{i}-1}\left|f_{i}(\tau, x(\tau))-f_{i}(\tau, 0)\right| \mathrm{d} \tau \\
\leq & \frac{1}{\Gamma(\alpha+1)}\left(L_{1} r+\mu\right)+\left(\theta_{1} L_{2}+\theta_{2} L_{3}\right) \frac{r}{\Gamma(\alpha)}+\sum_{i=1}^{m} \frac{L_{f} r+v_{i}}{\Gamma\left(\rho_{i}+1\right)} \leq r,
\end{aligned}
$$

which implies that $T B_{r} \subset B_{r}$.

Now, for $x, y \in X$ and for each $t \in J$, we obtain

$$
\begin{aligned}
|T x(t)-T y(t)| \leq & \sup _{t \in J}\left\{\frac{1}{\Gamma(\alpha)} \int_{0}^{t}(t-\tau)^{\alpha-1}|g(\tau, x(\tau), K x(\tau), H x(\tau))-g(\tau,(\tau), K x(\tau), H x(\tau))| \mathrm{d} \tau\right\} \\
& +\sum_{i=1}^{m} \frac{1}{\Gamma\left(\rho_{i}\right)} \int_{0}^{t}(t-\tau)^{\rho_{i}-1}\left|f_{i}(\tau, x(\tau))-f_{i}(\tau, y(\tau))\right| \mathrm{d} \tau \\
\leq & \left(\frac{L_{1}}{\Gamma(\alpha+1)}+\frac{\theta_{1} L_{2}+\theta_{2} L_{3}}{\Gamma(\alpha)}+\sum_{i=1}^{m} \frac{L_{f}}{\Gamma\left(\rho_{i}+1\right)}\right)\|x-y\| \leq R\|x-y\| .
\end{aligned}
$$


Consequently, we observe that $\|T x-T y\| \leq R\|x-y\|$. Since $R<1$, the operator $T$ is a contracting mapping. Hence, we conclude that the operator $T$ has a unique fixed point $x \in X$.

\section{Ulam Stability Results}

In this section, we will study the Ulam stability of problem (2). Let us consider the following inequality:

$$
\left|D^{\alpha}\left(x(t)-\sum_{i=1}^{m} I^{\rho_{i}} f_{i}(t, x(t))\right)-g(t, x(t), K x(t), H x(t))\right| \leq \varepsilon
$$

Definition 4. The Equation in (2) is Ulam-Hyers stable if there exists a real number $C_{f}>0$ such that for each $\varepsilon>0$ and for each solution $y \in C(J, \mathbb{R})$ of inequality (28), there exists a solution $x \in C(J, \mathbb{R})$ of equation (2) with

$$
|y(t)-x(t)| \leq \varepsilon C_{f}, \quad t \in J .
$$

Theorem 5. Assume that (H1) and (H3) are fulfilled. Then, problem (2) is Ulam-Hyers stable if $R<1$.

Proof. Let $\varepsilon>0$, and let $y \in C(J, \mathbb{R})$ be a function which satisfies inequality (28), and let $x \in C(J, \mathbb{R})$ be the unique solution of the following problem. Then, we recall that

$$
\begin{aligned}
x(t)= & \frac{1}{\Gamma(\alpha)} \int_{0}^{t}(t-\tau)^{\alpha-1} g(\tau, x(\tau), K x(\tau), H x(\tau)) \mathrm{d} \tau \\
& +\sum_{i=1}^{m} I^{\rho_{i}} f_{i}(t, x(t)) .
\end{aligned}
$$

Integrating inequality (28) and using the initial condition of problem (2), we get

$$
\left|y(t)-\sum_{i=1}^{m} I^{\rho_{i}} f_{i}(t, y(t))-\frac{1}{\Gamma(\alpha)} \int_{0}^{t}(t-\tau)^{\alpha-1} g(\tau, y(\tau), K y(\tau), H y(\tau)) \mathrm{d} \tau\right| \leq \frac{\varepsilon t^{\alpha}}{\Gamma(\alpha+1)}
$$

Now, we have

$$
\begin{aligned}
|y(t)-x(t)| \leq & \left|y(t)-\sum_{i=1}^{m} I^{\rho_{i}} f_{i}(t, y(t))-\frac{1}{\Gamma(\alpha)} \int_{0}^{t}(t-\tau)^{\alpha-1} g(\tau, y(\tau), K y(\tau), H y(\tau)) \mathrm{d} \tau\right| \\
& +\sum_{i=1}^{m} \frac{1}{\Gamma\left(\rho_{i}\right)} \int_{0}^{t}(t-\tau)^{\alpha-1}\left|f_{i}(t, y(t))-f_{i}(t, x(t))\right| \mathrm{d} \tau+\frac{1}{\Gamma(\alpha)} \int_{0}^{t}(t-\tau)^{\alpha-1} \\
& \times|g(\tau, y(\tau), K y(\tau), H y(\tau))-g(\tau, x(\tau), K x(\tau), H x(\tau))| \mathrm{d} \tau .
\end{aligned}
$$
obtain

Using hypothesis (H1) and (H3) and inequality (31), we

$$
\|y-x\| \leq \frac{\varepsilon}{\Gamma(\alpha+1)}+\left(\sum_{i=1}^{m} \frac{L_{f}}{\Gamma\left(\rho_{i}+1\right)}+\frac{L_{1}}{\Gamma(\alpha+1)}+\frac{\theta_{1} L_{2}+\theta_{2} L_{3}}{\Gamma(\alpha)}\right)\|y-x\|,
$$


and consequently we get

$$
\|y-x\| \leq \frac{\varepsilon}{\Gamma(\alpha+1)(1-R)}=\varepsilon C,
$$

where $C=(1 / \Gamma(\alpha+1)(1-R))$.

Thus, the considered problem (2) has the Ulam-Hyers stability.

\section{Illustrative Example}

In this section, an application of the results which have proved is provided. Let us consider Caputo fractional integro-differential equation as follows:

$$
\left\{\begin{array}{l}
D^{(3 / 2)}\left(x(t)-\sum_{i=1}^{2} I^{((3 i+2) / 3)} f_{i}(t, x(t))\right)=g(t, x(t), K x(t), H x(t)), \quad t \in J=[0,1], \\
x(0)=0, \\
D^{(3 / 2)} x(0)=\eta, \quad 1<\alpha<2,
\end{array}\right.
$$

where

$$
\begin{aligned}
f_{i}(t, x(t)) & =\frac{2 x(t)}{(2 i+t \sqrt{3})(23+i)}, \\
g(t, x(t), y(t), z(t)) & =\frac{|x(t)|}{100(1+|x(t)|)}+\frac{2 y(t)}{30\left(1+y^{2}(t)\right)}+\frac{2 z(t)}{55\left(1+z^{2}(t)\right)}-\cos t, \quad \forall t \in J ; x, y, z \in \mathbb{R} .
\end{aligned}
$$

Then, we have

$$
\left|f_{i}(t, x(t))-f_{i}(t, y(t))\right| \leq L_{f}|x-y| .
$$

An easy computation gives

$$
\begin{aligned}
& L_{f}=\frac{2}{2+\sqrt{3}}, \\
& L_{1}=\frac{1}{100}, \\
& L_{2}=\frac{1}{30}, \\
& L_{3}=\frac{1}{55}, \\
& \theta_{1}=\frac{\ln 2}{3}, \\
& \theta_{2}=\frac{\ln 2}{5} .
\end{aligned}
$$

Then, we have $R=\left(L_{1} / \Gamma(\alpha+1)\right)+\left(\theta_{1} L_{2}+\theta_{2} L_{3} / \Gamma(\alpha)\right)+$ $\sum_{i=1}^{m}\left(L_{f} / \Gamma\left(\rho_{i}+1\right)\right)=0.398<1$.

By Theorem 1, we see that problem (2) has a unique solution and has also the Ulam-Hyers stability.

\section{Conclusion}

In this work, we have considered a coupled VolterraFredholm integro-differential equation, and we have used the Caputo derivative operator. We prove two theorems and an example to illustrate our results. In the first theorem, we prove the existence and uniqueness of the solution, and the second theorem deals with the existence of at least one solution. The methods used here are Banach's fixed point theorem and Schaefer's fixed point theorem. Here, two Caputo derivative operators of different fractional orders were used in the considered equation, and it would be relevant to generalize this idea by considering several Caputo operators of different fractional orders. The example given on this work establishes the precision and efficiency of the proposed technique and shows that the problem has a unique solution. Before that, we have discussed the Ulam stability of the solution of problem (2).

\section{Data Availability}

No data were used to support this study.

\section{Conflicts of Interest}

The authors declare that they have no conflicts of interest.

\section{References}

[1] R. C. Calleja, A. R. Humphries, and B. Krauskopf, "Resonance phenomena in a scalar delay differential equation with two state-dependent delays," SIAM Journal on Applied Dynamical Systems, vol. 16, no. 3, Article ID 14741513, 2017.

[2] A. Carpinteri and F. Mainardi, Fractional Calculus in Continuum Mechanics, Springer, Berlin, Germany, 1997.

[3] D. Kumar, J. Singh, S. D. Purohit, and R. Swroop, "A hybrid analytical algorithm for nonlinear fractional wave-like 
equations," Mathematical Modelling of Natural Phenomena, vol. 14, no. 3, p. 304, 2019.

[4] Z. Dahmani and A. Taieb, "New existence and uniqueness results for high dimensional fractional differential systems," Facta Universitatis-Series: Mathematics and Informatics, vol. 30, no. 3, pp. 281-293, 2015.

[5] M. Feckan, J. Wang, and M. Pospísil, "Fractional-order equations and inclusions," Fractional Calculus in Applied Sciences and Engineering, De Gruyter, Berlin, Germany, 2017.

[6] Y. Gouari, Z. Dahmani, and A. Ndiaye, "A generalized sequential problem of Lane Emden type via fractional calculus," Moroccan Journal of Pure and Applied Analysis, vol. 6, no. 2, Article ID 168183, 2020.

[7] A. A. Kilbas, H. M. Srivastava, and J. J. Trujillo, Theory and Applications of Fractional Differential Equations, Elsevier B.V., Amsterdam, Netherlands, 2006.

[8] K. S. Miller and B. Ross, An Introduction to the Fractional Calculus and Fractional Differential Equations, Wiley, Hoboken, NJ, USA, 1993.

[9] N. Sene and A. Ndiaye, "On class of fractional-order chaotic or hyperchaotic systems in the context of the Caputo fractional-order derivative," Journal of Mathematics, vol. 2020, Article ID 8815377, 15 pages, 2020.

[10] A. Hamoud, "Existence and uniqueness of solutions for fractional neutral Volterra-Fredholm integro-differential equations," Advances in the Theory of Nonlinear Analysis and its Application, vol. 4, no. 4, pp. 321-331, 2020.

[11] A. Hamoud, N. Mohammed, and K. Ghadle, "Existence and uniqueness results for Volterra-Fredholm integro-differential equations," Advances in the Theory of Nonlinear Analysis and its Application, vol. 4, no. 4, pp. 361-372, 2020.

[12] A. Hamoud and K. Ghadle, "The approximate solutions of fractional Volterra-Fredholm integro-differential equations by using analytical techniques," Problemy Analiza-Issues of Analysis, vol. 7, no. 25, pp. 41-58, 2018.

[13] A. Hamoud and K. Ghadle, "Modified Laplace decomposition method for fractional Volterra-Fredholm integro-differential equations," Journal of Mathematical Modeling, vol. 6, no. 1, pp. 91-104, 2018.

[14] A. Hamoud and K. Ghadle, "Usage of the homotopy analysis method for solving fractional Volterra-Fredholm integrodifferential equation of the second kind," Tamkang Journal of Mathematics, vol. 49, no. 4, pp. 301-315, 2018.

[15] A. Hamoud and K. Ghadle, "Existence and uniqueness of solutions for fractional mixed Volterra-Fredholm integrodifferential equations," Indian Journal of Mathematics, vol. 60, no. 3, pp. 375-395, 2018.

[16] A. Hamoud, K. Ghadle, and S. Atshan, "The approximate solutions of fractional integro-differential equations by using modified Adomian decomposition method," Khayyam Journal of Mathematics, vol. 5, no. 1, pp. 21-39, 2019.

[17] K. Hattaf, "A new generalized definition of fractional derivative with non-singular kernel," Computation, vol. 8 , no. 2 , p. $49,2020$.

[18] A. Atangana and D. Baleanu, "New fractional derivatives with nonlocal and non-singular kernel: theory and application to heat transfer model," Thermal Science, vol. 20, no. 2, pp. 763-769, 2016.

[19] A. Caputo and M. Fabrizio, "A new definition of fractional derivative without singular kernel," Progress in Fractional Differentiation and Applications, vol. 1, pp. 73-85, 2015.

[20] S. Amiri, M. Hajipour, and D. Baleanu, "A spectral collocation method with piecewise trigonometric basis functions for nonlinear Volterra-Fredholm integral equations," Applied
Mathematics and Computation, vol. 370, Article ID 124915 , 2020.

[21] J. Wang, Y. Zhou, and M. Medved, "Existence and stability of fractional differential equations with Hadamard derivative," Topological Methods in Nonlinear Analysis, vol. 41, no. 1, pp. 113-133, 2013.

[22] B. Ahmad and S. Ntouyas, "Initial-value problems for hybrid Hadamard fractional differential equations," The Electronic Journal of Differential Equations, vol. 2014, no. 161, pp. 1-8, 2014.

[23] B. Ahmad, S. K. Ntouyas, and J. Tariboon, "A study of mixed Hadamard and Riemann-Liouville fractional integro-differential inclusions via endpoint theory," Applied Mathematics Letters, vol. 52, pp. 9-14, 2016.

[24] A. Hamoud, A. A. Charif, A. K. Ghadle, and K. P. Ghadle, "Existence and uniqueness results of hadamard fractional volterra-fredholm integro-differential equations," Konuralp Journal of Mathematics, vol. 9, no. 1, pp. 143-147, 2021.

[25] Y. Zhou, Basic Theory of Fractional Differential Equations, World Scientific, Singapore, 2014. 